\title{
Real-world treatment patterns, healthcare resource use and clinical outcomes of patients receiving second line therapy for advanced or metastatic gastric cancer
}

David Gómez-Ulloa ${ }^{1 *}$, Mayur Amonkar², Smita Kothari ${ }^{3}$, Winson Y. Cheung ${ }^{4}$, lan Chau ${ }^{5}$, John R. Zalcberg ${ }^{6}$, Núria Lara Suriñach ${ }^{1}$ and Alfredo Falcone ${ }^{7}$

\begin{abstract}
Background: Second-line (2 L) chemotherapies for advanced or metastatic gastric cancer have shown improved survival but there is no commonly accepted standard of care. This study examines real-world patient characteristics, treatment patterns, healthcare resource use (HCRU) and clinical outcomes in this setting.

Methods: Retrospective chart reviews were performed at participating institutions from Australia, Canada, Italy and UK for adult patients receiving $2 \mathrm{~L}$ treatment for advanced/metastatic disease from January 2013 to July 2015. Data were collected for 12 months or until death.

Results: Two hundred eighty patients were included, mean age was 60.9 years and 68.9\% were male. Half (51.8\%) received monotherapy in $2 \mathrm{~L}$, of whom $69.0 \%$ received taxanes. Irinotecan monotherapy was common in Australia (30.0\% of monotherapy patients) and Canada (43.8\%), but infrequent in Italy and UK. Doublet chemotherapy was used in $36.4 \%$ of $2 \mathrm{~L}$ patients, most commonly fluoropyrimidine + irinotecan. Use of targeted therapies (trastuzumab, ramucirumab) was infrequent except in Italy. Estimated median real-world progression-free survival (rwPFS) and real-world overall survival (rwOS) from the time of $2 \mathrm{~L}$ treatment initiation was 3.09 (95\% Cl: 2.76-3.68) and 6.54 (5.29-7.76) months, respectively, and estimated 12-month rwPFS and rwOS rate was 8 and 26\%, respectively. Only a minority (26.8\%) of patients were hospitalized during the follow-up period, with the lowest hospitalization in Italy (16.7\%). Laboratory and imaging tests were performed for 93.2 and $70.4 \%$, respectively.
\end{abstract}

Conclusions: About half of patients received monotherapy as $2 \mathrm{~L}$ chemotherapy for advanced/metastatic gastric cancer and a third received doublets. Real-world clinical outcomes for $2 \mathrm{~L}$ treatment are poor and HCRU is considerable.

Keywords: Gastric cancer, Chemotherapy, Outcomes, Health care utilization

\section{Background}

It is estimated that about 1 million people worldwide will be diagnosed with gastric cancer in 2018 [1]. It is the fifth most common cancer, accounting for $5-6 \%$ of all new cancer cases, but the third most prevalent cause

* Correspondence: david.gomez@iqvia.com

${ }^{1}$ Real World Solutions, IQVIA, Barcelona, Spain

Full list of author information is available at the end of the article of cancer mortality, contributing to $8-10 \%$ of cancer deaths $[1,2]$. The highest incidence and mortality rates are typically observed in East Asia and Central and Eastern Europe [1].

The prognosis for gastric cancer is generally poor. Because it is asymptomatic in the early stages, more than half of gastric adenocarcinomas, which comprise $80-85 \%$ of gastric cancers, are diagnosed at an advanced

(c) The Author(s). 2020 Open Access This article is licensed under a Creative Commons Attribution 4.0 International License, which permits use, sharing, adaptation, distribution and reproduction in any medium or format, as long as you give appropriate credit to the original author(s) and the source, provide a link to the Creative Commons licence, and indicate if changes were made. The images or other third party material in this article are included in the article's Creative Commons licence, unless indicated otherwise in a credit line to the material. If material is not included in the article's Creative Commons licence and your intended use is not permitted by statutory regulation or exceeds the permitted use, you will need to obtain permission directly from the copyright holder. To view a copy of this licence, visit http://creativecommons.org/licenses/by/4.0/. The Creative Commons Public Domain Dedication waiver (http://creativecommons.org/publicdomain/zero/1.0/) applies to the data made available in this article, unless otherwise stated in a credit line to the data. 
stage (locally advanced or metastatic) [3]. Systemic therapy remains the mainstay of treatment for patients with locally advanced and metastatic disease, and has been shown to improve survival, organ function and performance status compared with best supportive care (BSC) [4]. Currently available treatment options include platinums, irinotecan, epirubicin, fluoropyrimidines, and taxanes. Two targeted agents, trastuzumab and ramucirumab, are also approved in a few countries for the treatment of advanced or metastatic gastric cancer.

Until recently, there was a lack of robust evidence to support the use of salvage chemotherapy for advanced patients who progress after first-line $(1 \mathrm{~L})$ treatment. A number of randomized controlled trials have demonstrated the efficacy and feasibility of second-line $(2 \mathrm{~L})$ treatment for these patients. Both irinotecan and docetaxel as monotherapy are associated with significantly improved survival versus placebo [5-8]. However, no significant clinical benefit was shown for combination therapy with irinotecan plus cisplatin compared with irinotecan alone $[9,10]$. Use of ramucirumab in the $2 \mathrm{~L}$ setting has been shown to significantly improve survival, both as monotherapy versus placebo [11] and in combination with paclitaxel versus paclitaxel alone [12]. However, there was also evidence of increased grade 3 or worse leucopenia, neutropenia, anemia, hypertension, fatigue, and abdominal pain with the addition of ramucirumab to paclitaxel [12].

Despite these advances, therapeutic strategies for patients progressing after $1 \mathrm{~L}$ treatment are not clearly defined across clinical guidelines [13], and no consensus-driven standard of care has been defined. In the real-world setting, a number of studies have reported on $1 \mathrm{~L}$ treatment patterns and/or healthcare resource use (HCRU) in advanced or metastatic gastric cancer in Western countries [14-22]. However, evidence specific to patients who have progressed following $1 \mathrm{~L}$ treatment is limited. This article reports the results of GENERATE, an observational study on treatment patterns, HCRU and clinical outcomes of patients seen in routine clinical care who received $2 \mathrm{~L}$ therapy for advanced or metastatic gastric cancer. The study aims to improve the current understanding of the standard of care and the real-world patient outcomes in this setting using individual level data from four Western countries.

\section{Methods}

\section{Objectives}

The primary objectives of the GENERATE study were: 1) To describe the demographic and clinical characteristics of patients with metastatic or unresectable gastric or gastroesophageal junction (GEJ) cancer at initiation of 2 $\mathrm{L}$ anti-cancer systemic therapy after progression on previous therapy; 2) to describe treatment patterns in $2 \mathrm{~L}$ therapy in terms of drug regimen; 3) to describe HCRU during $2 \mathrm{~L}$ therapy, including management of cancer and adverse events (AEs), and biomarker testing. Secondary objectives were to assess real-world progression-free survival (rwPFS) and real-world overall survival (rwOS) from the start of $2 \mathrm{~L}$ therapy, both overall and by type of therapy, and to describe the factors associated with these outcomes.

\section{Study design}

GENERATE was an observational, retrospective chart review study of a single pooled cohort which included patients with confirmed metastatic or unresectable gastric or GEJ adenocarcinoma who initiated $2 \mathrm{~L}$ drug therapy in Australia, Canada, Italy and the United Kingdom (UK). The investigators were medical oncologists, radiation oncologists and/or surgeons who specialized in gastrointestinal cancer management. Patients were identified starting from those who initiated $2 \mathrm{~L}$ therapy at the participating institutions in July 2015, and working backwards until an a priori target of 100 patients were identified for each country, or until January 2013. The index date was the day that the patient started $2 \mathrm{~L}$ therapy. Each patient's chart was reviewed for the 12 months following the index date or until death, whichever occurred first; this was defined as the observation period.

Patients were included if they were aged $\geq 18$ years at index date, had a diagnosis of metastatic or unresectable gastric or GEJ adenocarcinoma confirmed through histology and/or cytology, had a full medical history available at the participating site for the follow-up period, provided written informed consent (where required under national or institutional policies), were not participating in any interventional clinical trials during the follow-up period, and did not present with any other prior or concomitant malignancy, with the exception of basal cell carcinoma and squamous cell carcinoma of the skin, which were not excluded.

The study protocol was approved by the appropriate national or local ethics committee or institutional review board for each study site. Informed consent was collected for patients from Italy who were alive at the time of data abstraction. In the other countries, the need for informed consent was waived by the ethics committee due to the retrospective nature of the study and the minimal risk to patients. Demographic and clinical variables, gastric or GEJ cancer history and $1 \mathrm{~L}$ treatment were collected at the index date. Data that were collected for the entire follow-up period included details of $2 \mathrm{~L}$ and 3 L therapy like regimen, reason for discontinuation (if progression was stated, date of progression was defined as the date when treatment line was stopped), treatment outcomes (current or last available clinical status), and 
HCRU (hospitalizations, outpatient and emergency room visits, and laboratory and imaging tests performed).

All analyses were performed by country and for the overall study population. Since no hypothesis was tested, only descriptive statistics were employed. Additionally, Kaplan-Meier curves were used to present rwPFS and rwOS, and a multivariate model was used to explore the associations of covariates with rwPFS and rwOS. Patients with missing ECOG performance status were excluded from the final model.

\section{Results}

\section{Baseline characteristics and $1 \mathrm{~L}$ treatment}

Overall, 280 patients were included in the study: 34 from Australia (12.2\%, from 6 sites), 100 from Canada (37.5\%, from 6 sites), 84 from Italy (30.0\%, from 8 sites), and 62 from the UK (22.1\%, from 5 sites). Of these, 60 completed the 12-month observation period, 199 died before the end of the observation period, and 21 (of whom 14 were from Italy) were lost to follow-up. The majority of patients were male (68.9\%), and Caucasian $(84.9 \%$ of those with ethnicity recorded), and mean age was 60.9 $( \pm 12.0)$ years.

Almost all patients $(93.6 \%)$ had received combination chemotherapy as $1 \mathrm{~L}$ treatment, of whom $67.9 \%$ had received triplet chemotherapy, most commonly anthracycline added to a fluoropyrimidine + platinum doublet. Approximately three-quarters (76.1\%) of patients had undergone human epidermal growth factor receptor 2 (HER2) testing, of whom approximately one in five (22.0\%) tested positive, and the addition of the antiHER2 agent trastuzumab to the $1 \mathrm{~L}$ chemotherapy regimen was reported for $65.2 \%$ of these patients. Additional details regarding baseline patient characteristics, biomarker testing and $1 \mathrm{~L}$ treatment are shown in Table 1.

\section{$2 \mathrm{~L}$ treatment patterns}

All patients initiated $2 \mathrm{~L}$ therapy, given that this was an inclusion criterion for the study. The treatments used are shown in Table 2; specific drug combinations for $2 \mathrm{~L}$ and $3 \mathrm{~L}$ therapy are described in greater detail in the supplementary material (Tables s1 and s2). Approximately half of patients $(51.8 \%)$ received monotherapy, with no major differences in this proportion between countries. Taxanes (paclitaxel or docetaxel) were the most common monotherapy agents, used by $69.0 \%$ of all patients receiving monotherapy. However, there were considerable differences between countries: in the UK, $97.4 \%$ of monotherapy patients received taxanes, compared with only half in Australia and Canada. Irinotecan was used in $43.8 \%$ of Canadian monotherapy patients and $30.0 \%$ of those in Australia, but its use was limited in Italy and the UK. Few patients in any country received other monotherapies.
There was more heterogeneity in the type of combination regimens used. Doublet chemotherapy was used in $36.4 \%$ of patients overall and $75.6 \%$ of patients receiving combination therapy. A third of patients on combination chemotherapy received fluoropyrimidine + irinotecan, and $17.8 \%$ received fluoropyrimidine + platinum. A small proportion received a taxane in combination with a novel agent (ramucirumab in $9.6 \%$ of patients receiving combination therapy and trastuzumab in $3.7 \%$ ); nearly all of these cases were those treated in Italy. Almost a quarter of patients who received combination therapy (23.7\%) were treated with triplet chemotherapy; this was almost always based on fluoropyrimidine + platinum, with either anthracycline or another agent added.

\section{$3 \mathrm{~L}$ treatment patterns}

Overall, fewer than a third of patients (29.6\%) received 3 L chemotherapy, and the proportion was largely similar between countries (Table 3). Roughly equal proportions received monotherapy and combination therapy, except in Canada where combination therapy was less common in $3 \mathrm{~L}$. As in $2 \mathrm{~L}$, taxanes were the most commonly used monotherapy, followed by irinotecan. Few patients received monotherapy with novel agents. Most patients who had combination therapy received a doublet, most commonly fluoropyrimidines in combination with irinotecan or platinum; triplet therapy was uncommon.

Patients' transition from $1 \mathrm{~L}$ to $2 \mathrm{~L}$ and $3 \mathrm{~L}$ of treatment is shown in Fig. 1 as a Sankey Diagram of the most common therapies that were used in the next line following specific therapies in the previous line. There was a general trend for patients to proceed from a fluoropyrimidine/platinum/anthracycline triplet or a fluoropyrimidine/platinum doublet in $1 \mathrm{~L}$, to $2 \mathrm{~L}$ treatment based on irinotecan or taxanes as monotherapy or a fluoropyrimidine/irinotecan doublet, followed by $3 \mathrm{~L}$ treatment consisting of taxane monotherapy or fluoropyrimidine/ irinotecan doublet regimens.

\section{Real-world treatment outcomes}

Median rwPFS for patients initiating $2 \mathrm{~L}$ treatment was estimated to be 3.09 (95\% CI: 2.76-3.68) months from the date of initiation of therapy. The estimated probability of rwPFS at 3, 6, 9 and 12 months was $51,27,14$ and $8 \%$, respectively. Median (95\% CI) rwOS was estimated to be 6.54 (5.29-7.76) months, and the estimated probability of rwOS at $6,12,18$ and 24 months after treatment initiation was 53,26, 15 and 5\%, respectively.

When evaluated according to treatment regimen, both rwPFS and rwOS showed notable differences (see Figs. 2 and 3). Median rwPFS increased from 2.69 (95\% CI 2.11-2.86) months with monotherapy to 3.43 (95\% CI 2.73-3.91) months with doublet chemotherapy (statistical significance not reached), and 7.95 (95\% CI $4.43-$ 
Table 1 Baseline sociodemographic and clinical characteristics, and $1 \mathrm{~L}$ treatment

\begin{tabular}{|c|c|c|c|c|c|}
\hline & Australia $(N=34)$ & Canada $(N=100)$ & Italy $(N=84)$ & UK $(N=62)$ & Overall $(N=280)$ \\
\hline \multicolumn{6}{|l|}{ General demographic and clinical characteristics } \\
\hline Male gender, n (\%) & $22(64.7 \%)$ & $68(68.0 \%)$ & $61(72.6 \%)$ & $42(67.7 \%)$ & $193(68.9 \%)$ \\
\hline \multicolumn{6}{|l|}{ Age (years) } \\
\hline Mean (SD) & $65.7(10.5)$ & $60.7(11.0)$ & $60.6(11.9)$ & $58.8(14.0)$ & $60.9(12.0)$ \\
\hline Range & $42-83$ & $33-85$ & $34-83$ & $26-81$ & $26-85$ \\
\hline \multicolumn{6}{|l|}{ Ethnicity, n (\%) } \\
\hline Caucasian & $32(94.1 \%)$ & $37(60.7 \%)$ & $84(100.0 \%)$ & $44(83.0 \%)$ & $197(84.9 \%)$ \\
\hline Asian & $2(5.9 \%)$ & $11(18.0 \%)$ & - & $4(7.5 \%)$ & $17(7.3 \%)$ \\
\hline Other & - & $13(21.3 \%)$ & - & $5(9.4 \%)$ & $18(7.8 \%)$ \\
\hline Unknown/missing & - & 39 & - & 9 & 48 \\
\hline \multicolumn{6}{|l|}{ Smoking status, n (\%) } \\
\hline Current smoker & $6(23.1 \%)$ & $13(13.7 \%)$ & $11(14.5 \%)$ & $7(14.6 \%)$ & $37(15.1 \%)$ \\
\hline Ex-smoker & $9(34.6 \%)$ & $38(40.0 \%)$ & $37(48.7 \%)$ & $22(45.8 \%)$ & $106(43.3 \%)$ \\
\hline Non-smoker & $11(42.3 \%)$ & $44(46.3 \%)$ & $28(36.8 \%)$ & 19 (39.6\%) & $102(41.6 \%)$ \\
\hline Unknown/missing & 8 & 5 & 8 & 14 & 35 \\
\hline \multicolumn{6}{|l|}{ Alcohol consumption, $\mathrm{n}(\%)^{\mathrm{a}}$} \\
\hline Heavy & $1(4.8 \%)$ & $6(6.7 \%)$ & $2(3.5 \%)$ & $3(7.0 \%)$ & $12(5.7 \%)$ \\
\hline Occasional & $11(52.4 \%)$ & $53(59.6 \%)$ & $37(64.9 \%)$ & $31(72.1 \%)$ & $132(62.9 \%)$ \\
\hline Abstinent & $9(42.9 \%)$ & $30(33.7 \%)$ & $18(31.6 \%)$ & 9 (20.9\%) & $66(31.4 \%)$ \\
\hline Unknown/missing & 13 & 11 & 27 & 19 & 70 \\
\hline \multicolumn{6}{|l|}{ Comorbidities, n (\%) } \\
\hline None & $8(23.5 \%)$ & $31(31.0 \%)$ & $42(50.0 \%)$ & $21(33.9 \%)$ & $102(36.4 \%)$ \\
\hline Hypertension & $13(38.2 \%)$ & 29 (29.0\%) & $23(27.4 \%)$ & $24(38.7 \%)$ & $89(31.8 \%)$ \\
\hline Diabetes mellitus & $4(11.8 \%)$ & $12(12.0 \%)$ & $11(13.1 \%)$ & $9(14.5 \%)$ & $36(12.9 \%)$ \\
\hline Dyslipidemia & $7(20.6 \%)$ & $14(14.0 \%)$ & $6(7.1 \%)$ & $6(9.7 \%)$ & $33(11.8 \%)$ \\
\hline GI disease (other than gastric or GEJ cancer) & $3(8.8 \%)$ & $11(11.0 \%)$ & $6(7.1 \%)$ & $3(4.8 \%)$ & $23(8.2 \%)$ \\
\hline Cardiovascular disease & $5(14.7 \%)$ & $13(13.0 \%)$ & $7(8.3 \%)$ & $5(8.1 \%)$ & $30(10.7 \%)$ \\
\hline \multicolumn{6}{|l|}{ Disease history and characteristics } \\
\hline Time since diagnosis (months), median (range) & $12.01(5.09-43.41)$ & $7.79(1.64-112.88)$ & $9.91(2.83-102.83)$ & $9.76(2.33-61.78)$ & $9.40(1.64-112.88)$ \\
\hline \multicolumn{6}{|l|}{ Location of metastasis, $\mathrm{n}(\%)^{c}$} \\
\hline None & $2(5.9 \%)$ & $6(6.0 \%)$ & $3(3.6 \%)$ & $3(4.8 \%)$ & $14(5.0 \%)$ \\
\hline Peritoneal & $10(29.4 \%)$ & $34(34.0 \%)$ & $32(38.1 \%)$ & $18(29.0 \%)$ & $94(33.6 \%)$ \\
\hline Liver & $21(61.8 \%)$ & $40(40.0 \%)$ & $33(39.3 \%)$ & $24(38.7 \%)$ & $118(42.1 \%)$ \\
\hline Bone & $3(8.8 \%)$ & $13(13.0 \%)$ & $11(13.1 \%)$ & $5(8.1 \%)$ & $32(11.4 \%)$ \\
\hline Lung & $7(20.6 \%)$ & $23(23.0 \%)$ & $20(23.8 \%)$ & $9(14.5 \%)$ & $59(21.1 \%)$ \\
\hline Other & $11(32.4 \%)$ & $46(46.0 \%)$ & $30(35.7 \%)$ & $26(41.9 \%)$ & $113(40.4 \%)$ \\
\hline \multicolumn{6}{|l|}{ Number of metastatic locations, n (\%) } \\
\hline 1 & $14(43.8 \%)$ & $39(41.5 \%)$ & $46(56.8 \%)$ & $31(52.5 \%)$ & $130(48.9 \%)$ \\
\hline 2 & $8(25.0 \%)$ & $30(31.9 \%)$ & $14(17.3 \%)$ & $14(23.7 \%)$ & $66(24.8 \%)$ \\
\hline 3 & $6(18.8 \%)$ & $14(14.9 \%)$ & $14(17.3 \%)$ & $7(11.9 \%)$ & $41(15.4 \%)$ \\
\hline $4+$ & $4(12.5 \%)$ & $11(11.7 \%)$ & $7(8.6 \%)$ & $7(11.9 \%)$ & $29(10.9 \%)$ \\
\hline \multicolumn{6}{|l|}{ ECOG PS at initiation of $2 \mathrm{~L}$ treatment, $\mathrm{n}(\%)$} \\
\hline Unknown & $9(26.5 \%)$ & $32(32.0 \%)$ & $9(10.7 \%)$ & 5 (8.1\%) & 55 (19.6\%) \\
\hline 0 & 7 (28.0\%) & 7 (10.3\%) & $19(25.3 \%)$ & $12(21.1 \%)$ & 45 (20.0\%) \\
\hline
\end{tabular}


Table 1 Baseline sociodemographic and clinical characteristics, and $1 \mathrm{~L}$ treatment (Continued)

\begin{tabular}{|c|c|c|c|c|c|}
\hline & Australia $(N=34)$ & Canada $(N=100)$ & Italy $(N=84)$ & UK $(N=62)$ & Overall $(N=280)$ \\
\hline 1 & $17(68.0 \%)$ & $41(60.3 \%)$ & $45(60.0 \%)$ & $38(66.7 \%)$ & $141(62.7 \%)$ \\
\hline 2 & $1(4.0 \%)$ & $18(26.5 \%)$ & $11(14.7 \%)$ & $5(8.8 \%)$ & $35(15.6 \%)$ \\
\hline 3 & - & $2(2.9 \%)$ & - & $2(3.5 \%)$ & $4(1.8 \%)$ \\
\hline \multicolumn{6}{|l|}{ HER2 test, n (\%) } \\
\hline Not performed & $20(58.8 \%)$ & $28(28.0 \%)$ & $14(16.7 \%)$ & $5(8.1 \%)$ & $67(23.9 \%)$ \\
\hline Performed & $14(41.2 \%)$ & $72(72.0 \%)$ & $70(83.3 \%)$ & 57 (91.9\%) & $213(76.1 \%)$ \\
\hline Performed prior to $1 \mathrm{~L}$ initiation & $8(61.5 \%)$ & $50(76.9 \%)$ & $60(87.0 \%)$ & $46(85.2 \%)$ & $164(81.6 \%)$ \\
\hline Performed later in treatment & $5(38.5 \%)$ & $15(23.1 \%)$ & $9(13.0 \%)$ & $8(14.8 \%)$ & 37 (18.4\%) \\
\hline HER2 test positive ${ }^{d}$ & $3(21.4 \%)$ & $17(24.6 \%)$ & $15(21.4 \%)$ & $11(19.6 \%)$ & $46(22.0 \%)$ \\
\hline HER2 test negative ${ }^{d}$ & $11(78.6 \%)$ & $52(75.4 \%)$ & $55(78.6 \%)$ & 45 (80.4\%) & $163(78.0 \%)$ \\
\hline HER2 test result not available & - & 3 & - & 1 & 4 \\
\hline History of surgery ${ }^{\mathrm{e}}$ & $8(24.2 \%)$ & $15(15.5 \%)$ & $15(18.3 \%)$ & $4(6.6 \%)$ & $42(15.4 \%)$ \\
\hline History of radiotherapy ${ }^{f}$ & $4(12.9 \%)$ & $25(25.5 \%)$ & $2(2.5 \%)$ & $8(13.3 \%)$ & $39(14.5 \%)$ \\
\hline \multicolumn{6}{|l|}{$1 \mathrm{~L}$ anti-cancer drug therapy, $\mathrm{n}(\%)^{\mathrm{g}}$} \\
\hline Monotherapy & $3(8.8 \%)$ & $3(3.0 \%)$ & $9(10.7 \%)$ & $3(4.8 \%)$ & $18(6.4 \%)$ \\
\hline Fluoropyrimidine & $1(33.3 \%)$ & $3(100.0 \%)$ & $4(44.4 \%)$ & - & $8(44.4 \%)$ \\
\hline Taxane & $1(33.3 \%)$ & - & $4(44.4 \%)$ & $3(100.0 \%)$ & $8(44.4 \%)$ \\
\hline Platinum & - & - & $1(11.1 \%)$ & - & $1(5.6 \%)$ \\
\hline Other monotherapy & $1(33.3 \%)$ & - & - & - & $1(5.6 \%)$ \\
\hline Combination therapy & $31(91.2 \%)$ & 97 (97.0\%) & 75 (89.3\%) & $59(95.2 \%)$ & $262(93.6 \%)$ \\
\hline Doublet chemotherapy & $7(22.6 \%)$ & $25(25.8 \%)$ & $30(40.0 \%)$ & $12(20.3 \%)$ & $74(28.2 \%)$ \\
\hline Fluoropyrimidine + platinum & $7(22.6 \%)$ & $22(22.7 \%)$ & $25(33.3 \%)$ & $11(18.6 \%)$ & $65(24.8 \%)$ \\
\hline Fluoropyrimidine + Irinotecan & - & $1(1.0 \%)$ & $3(4.0 \%)$ & - & $4(1.5 \%)$ \\
\hline Other doublet & - & $2(2.1 \%)$ & $2(2.7 \%)$ & $1(1.7 \%)$ & $5(1.9 \%)$ \\
\hline Triplet chemotherapy & $22(71.0 \%)$ & $71(73.2 \%)$ & $42(56.0 \%)$ & $43(72.9 \%)$ & $178(67.9 \%)$ \\
\hline Fluoropyrimidine + platinum + anthracycline & $21(67.7 \%)$ & $64(66.0 \%)$ & $18(24.0 \%)$ & $31(52.5 \%)$ & $134(51.1 \%)$ \\
\hline Fluoropyrimidine + platinum + other & $1(3.2 \%)$ & $7(7.2 \%)$ & $23(30.7 \%)$ & $12(20.3 \%)$ & $43(16.4 \%)$ \\
\hline Other triplet & - & - & $1(1.3 \%)$ & - & $1(0.4 \%)$ \\
\hline
\end{tabular}

${ }^{a}$ Heavy: $>3$ (women) or $>4$ (men) standard drinks/day; Occasional: $<3$ or $<4$ standard drinks/day, respectively; Abstinent $=$ never drinks alcohol

${ }^{\mathrm{b}}$ Only comorbidities present in $\geq 5 \%$ of the overall study population are listed

c Multiple responses were allowed

${ }^{\mathrm{d}}$ Out of patients who had test; information on test was missing/unkown in 12 patients (7 from Canada, 1-3 from other countries), result was missing/unknown in 3 patients from Canada and 1 from the UK

e Surgical treatment of gastric/GEJ cancer; data were missing/unknown in 7 patients (1-3 in each country)

${ }^{\mathrm{f}}$ Includes neoadjuvant and palliative radiotherapy; data were missing/unknown in 11 patients (2-4 in each country)

${ }^{g}$ Percentages for individual regimens are out of number of patients who received monotherapy or combination therapy, respectively

$1 \mathrm{~L}$ first line, ECOG Eastern Cooperative Oncology Group, GEJ gastroesophageal junction, Gl gastrointestinal, HER2 human epidermal growth factor receptor 2, SD

standard deviation

10.25) with triplet chemotherapy $(p<0.001$, Log-rank test). Similarly, median rwOS increased from 4.50 (95\% CI 3.81-5.52) months with monotherapy to 7.53 (95\% CI 6.41-8.74) months with doublet chemotherapy and 14.66 (95\% CI 9.13-18.56) with triplet chemotherapy ( $\mathrm{p}<0.001$, Log-rank test).

In terms of survival with individual $2 \mathrm{~L}$ treatment regimens, taxane monotherapy and irinotecan monotherapy showed similar rwPFS (median 2.74 [95\% CI 2.00-3.35] and 2.10 [95\% CI 1.05-2.79] months, respectively) (Fig. 2), but rwOS with taxanes was slightly longer (median 4.73 [95\% CI 3.88-6.05] versus 3.70 [95\% CI 2.17-5.03]) (Fig. 3). Of the most common doublet therapies, PFS was numerically longer with fluoropyrimidine + platinum (5.44 [95\% CI 2.76-7.66] months) than with fluoropyrimidine + irinotecan (3.09 [95\% CI 2.53-3.78] months). This translated into numerically longer rwOS with fluoropyrimidine + platinum (9.04 [7.00-12.52] versus 6.54 [4.57-7.99] months). 
Table $22 \mathrm{~L}$ chemotherapy

\begin{tabular}{|c|c|c|c|c|c|}
\hline Therapy, n (\%) & Australia $(N=34)$ & Canada $(N=100)$ & Italy $(N=84)$ & UK $(N=62)$ & Overall $(N=280)$ \\
\hline Monotherapy & $20(58.8 \%)$ & $48(48.0 \%)$ & $38(45.2 \%)$ & $39(62.9 \%)$ & $145(51.8 \%)$ \\
\hline Combination therapy ${ }^{\mathrm{b}}$ & $14(41.2 \%)$ & $52(52.0 \%)$ & $46(54.8 \%)$ & $23(37.1 \%)$ & $135(48.2 \%)$ \\
\hline Doublet chemotherapy & $10(71.4 \%)$ & 39 (75.0\%) & $38(82.6 \%)$ & $15(65.2 \%)$ & $102(75.6 \%)$ \\
\hline Triplet chemotherapy & $4(28.6 \%)$ & $13(25.0 \%)$ & $8(17.4 \%)$ & 7 (30.4\%) & $32(23.7 \%)$ \\
\hline \multicolumn{6}{|l|}{ Monotherapy $^{c}$} \\
\hline Taxanes & $10(50.0 \%)$ & $23(47.9 \%)$ & $29(76.3 \%)$ & 38 (97.4\%) & $100(69.0 \%)$ \\
\hline Irinotecan & $6(30.0 \%)$ & $21(43.8 \%)$ & $4(10.5 \%)$ & $1(2.6 \%)$ & $32(22.1 \%)$ \\
\hline Fluoropyrimidines & $2(10.0 \%)$ & $3(6.3 \%)$ & $1(2.6 \%)$ & - & $6(4.1 \%)$ \\
\hline Ramucirumab & - & $1(2.1 \%)$ & $3(7.9 \%)$ & - & $4(2.8 \%)$ \\
\hline Platinum & $1(5.0 \%)$ & - & $1(2.6 \%)$ & - & $2(1.4 \%)$ \\
\hline Other monotherapy & $1(5.0 \%)$ & - & - & - & $1(0.7 \%)$ \\
\hline \multicolumn{6}{|l|}{ Doublet chemotherapy ${ }^{c}$} \\
\hline Fluoropyrimidine + irinotecan & $2(14.3 \%)$ & $25(48.1 \%)$ & $18(39.1 \%)$ & - & $45(33.3 \%)$ \\
\hline Fluoropyrimidine + platinum & $5(35.7 \%)$ & $6(11.5 \%)$ & $4(8.7 \%)$ & $9(39.1 \%)$ & $24(17.8 \%)$ \\
\hline Taxanes + ramucirumab & - & $2(3.8 \%)$ & $10(21.7 \%)$ & $1(4.3 \%)$ & $13(9.6 \%)$ \\
\hline Taxanes + trastuzumab & - & - & $3(6.5 \%)$ & $2(8.7 \%)$ & $5(3.7 \%)$ \\
\hline Other doublet & $3(21.4 \%)$ & $6(11.5 \%)$ & $3(6.5 \%)$ & $3(13.0 \%)$ & $15(11.1 \%)$ \\
\hline \multicolumn{6}{|l|}{ Triplet chemotherapy ${ }^{c}$} \\
\hline Fluoropyrimidine + platinum + anthracycline & $4(28.6 \%)$ & $5(9.6 \%)$ & $3(6.5 \%)$ & $3(13.0 \%)$ & $15(11.1 \%)$ \\
\hline Fluoropyrimidine + platinum + other & - & $8(15.4 \%)$ & $5(10.9 \%)$ & $2(8.7 \%)$ & $15(11.1 \%)$ \\
\hline Other triplet & - & - & - & 2 (8.7\%) & $2(1.5 \%)$ \\
\hline
\end{tabular}

a Specific drugs used are tabulated in the supplementary material

b Percentages receiving doublet and triplet are out of number of patients who received combination therapy

${ }^{c}$ Percentages for individual regimens are out of number of patients who received monotherapy or combination therapy, respectively

In the multivariate analysis of rwOS, use of combination therapy in $2 \mathrm{~L}$ was associated with a trend for longer survival compared with monotherapy (HR for death 0.738 [95\% CI 0.51-1.08], $p=0.114$ ). Factors associated with shorter survival were ECOG performance status $>1$ at $2 \mathrm{~L}$ treatment initiation (HR 1.50 [95\% CI 0.94-2.40], $p=0.090$ ), and presence of more than one metastatic location (HR 1.58 [95\% CI 1.07-2.32], $p=0.010$ ). However, only the association with number of metastatic locations reached statistical significance. Presence of $>1$ metastatic location was also significantly associated with shorter PFS (HR 1.51 [95\% CI 1.048-2.172], $p=0.027$ ). There were non-significant associations between female gender and shorter rwPFS, and combination therapy and longer rwPFS. Results for the multivariate analyses are shown in the supplementary material (Table s3 and Table s4).

\section{Healthcare resource use}

The number and percentage of patients using each healthcare resource during $2 \mathrm{~L}$ treatment, regardless of reason for the use, is shown in Table 4. Average followup period was 6.6 months. Only a minority $(26.8 \%)$ of patients were hospitalized (hospitalization may have been same-day), with hospitalization least likely in Italy (16.7\%, versus $30.0-32.4 \%$ elsewhere). Intensive care unit (ICU) stays were rare. Almost all patients (94.6\%) received concomitant medications. Laboratory tests and imaging studies were performed for 93.2 and $70.4 \%$ of patients, respectively. Patients in Italy were markedly less likely to undergo imaging tests $(44.0 \%$, versus $76.5-$ 90.3\% elsewhere).

\section{Discussion}

The GENERATE study provides a detailed description of patient characteristics, treatment patterns, HCRU and survival outcomes for real-world patients initiating $2 \mathrm{~L}$ treatment for advanced or metastatic gastric or GEJ adenocarcinoma who were seen in routine clinical practice across four different Western countries.

The sociodemographic and clinical characteristics of patients included in GENERATE were broadly comparable with patients in randomized controlled trials (RCTs). In terms of age, patients included in GENERATE were similar to participants included in the most recent RCTs evaluating $2 \mathrm{~L}$ treatment $[7,8,10-12,23$, 
Table $33 \mathrm{~L}$ chemotherapy

\begin{tabular}{|c|c|c|c|c|c|}
\hline Therapy, n (\%) & Australia ( $N=9)$ & Canada $(N=37)$ & Italy $(N=19)$ & UK $(N=18)$ & Overall $(N=83)$ \\
\hline Monotherapy & $4(44.4 \%)$ & $28(75.7 \%)$ & $10(52.6 \%)$ & $10(55.6 \%)$ & $52(62.7 \%)$ \\
\hline Combination therapy ${ }^{\mathrm{b}}$ & $5(55.6 \%)$ & $9(24.3 \%)$ & $9(47.4 \%)$ & $8(44.4 \%)$ & $31(37.3 \%)$ \\
\hline Doublet chemotherapy & $5(100.0 \%)$ & $7(77.8 \%)$ & $8(88.9 \%)$ & $6(85.7 \%)$ & $26(86.7 \%)$ \\
\hline Triplet chemotherapy & - & $2(22.2 \%)$ & $1(11.1 \%)$ & $1(14.3 \%)$ & $4(13.3 \%)$ \\
\hline Missing & - & - & - & 1 & 1 \\
\hline \multicolumn{6}{|l|}{ Monotherapyc } \\
\hline Taxanes & $3(75.0 \%)$ & $18(64.3 \%)$ & $8(80.0 \%)$ & $7(70.0 \%)$ & $36(69.2 \%)$ \\
\hline Irinotecan & $1(25.0 \%)$ & $8(28.6 \%)$ & - & $1(10.0 \%)$ & $10(19.2 \%)$ \\
\hline Ramucirumab & - & $1(3.6 \%)$ & $1(10.0 \%)$ & $1(10.0 \%)$ & $3(5.8 \%)$ \\
\hline Fluoropyrimidines & - & $1(3.6 \%)$ & $1(10.0 \%)$ & - & $2(3.8 \%)$ \\
\hline Other monotherapy & - & - & - & $1(10.0 \%)$ & $1(1.9 \%)$ \\
\hline \multicolumn{6}{|l|}{ Doublet chemotherapy ${ }^{c}$} \\
\hline Fluoropyrimidine + irinotecan & $5(100.0 \%)$ & $2(22.2 \%)$ & $4(44.4 \%)$ & $5(71.4 \%)$ & $16(53.3 \%)$ \\
\hline Fluoropyrimidine + platinum & - & $3(33.3 \%)$ & $3(33.3 \%)$ & - & $6(20.0 \%)$ \\
\hline Taxanes + ramucirumab & - & $1(11.1 \%)$ & $1(11.1 \%)$ & $1(14.3 \%)$ & $3(10.0 \%)$ \\
\hline Other doublet & - & $1(11.1 \%)$ & - & - & $1(3.3 \%)$ \\
\hline \multicolumn{6}{|l|}{ Triplet chemotherapy ${ }^{c}$} \\
\hline Fluoropyrimidine + platinum + anthracycline & - & $1(11.1 \%)$ & - & - & $1(3.3 \%)$ \\
\hline Fluoropyrimidine + platinum + other & - & $1(11.1 \%)$ & $1(11.1 \%)$ & - & $2(6.7 \%)$ \\
\hline Other triplet & - & - & - & $1(14.3 \%)$ & $1(3.3 \%)$ \\
\hline
\end{tabular}

${ }^{a}$ Specific drugs used are tabulated in the supplementary material

${ }^{\mathrm{b}}$ Percentages receiving doublet and triplet are out of number of patients who received combination therapy

c Percentages for individual regimens are out of number of patients who received monotherapy, doublet or triplet therapy, respectively

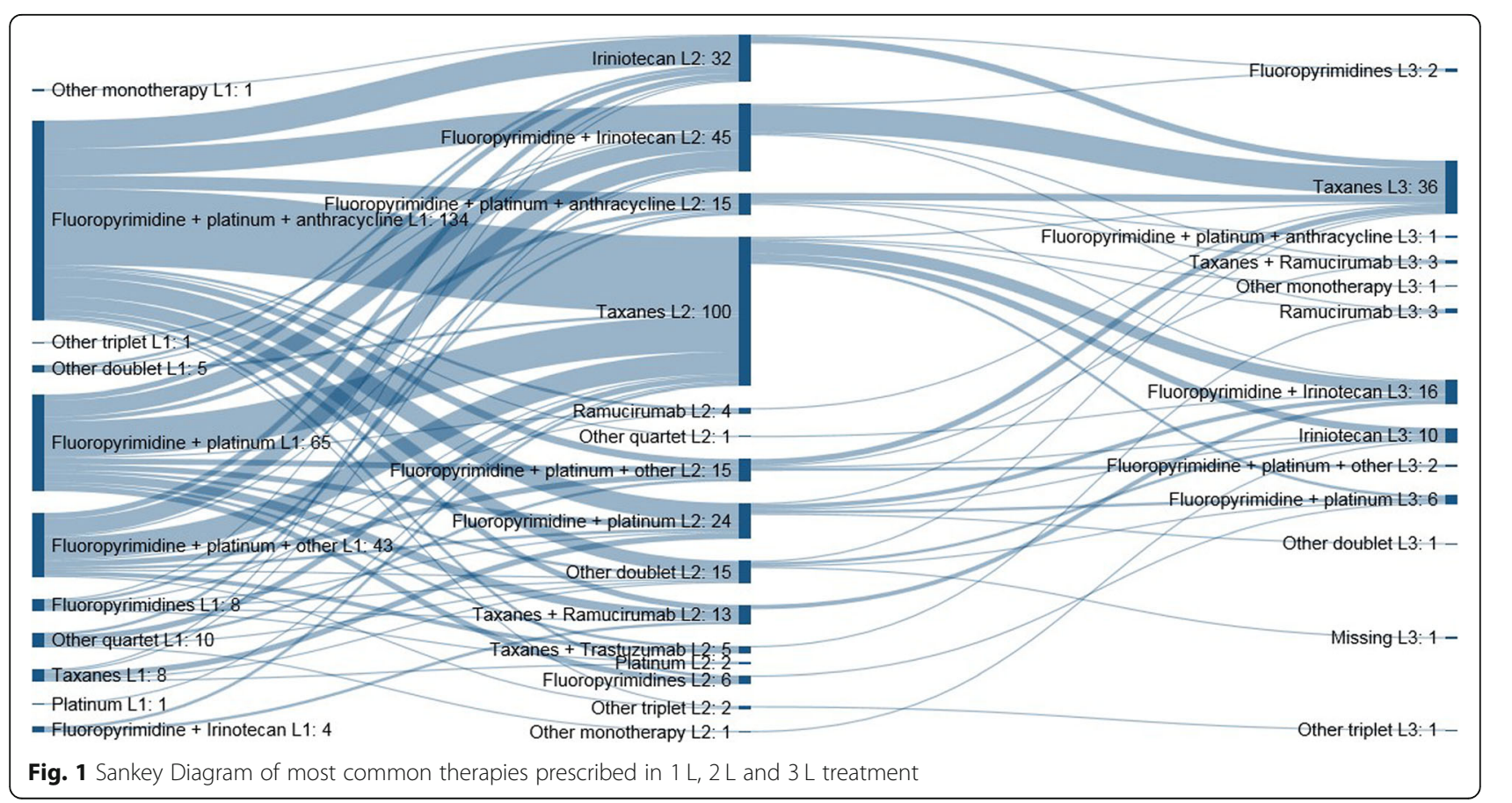




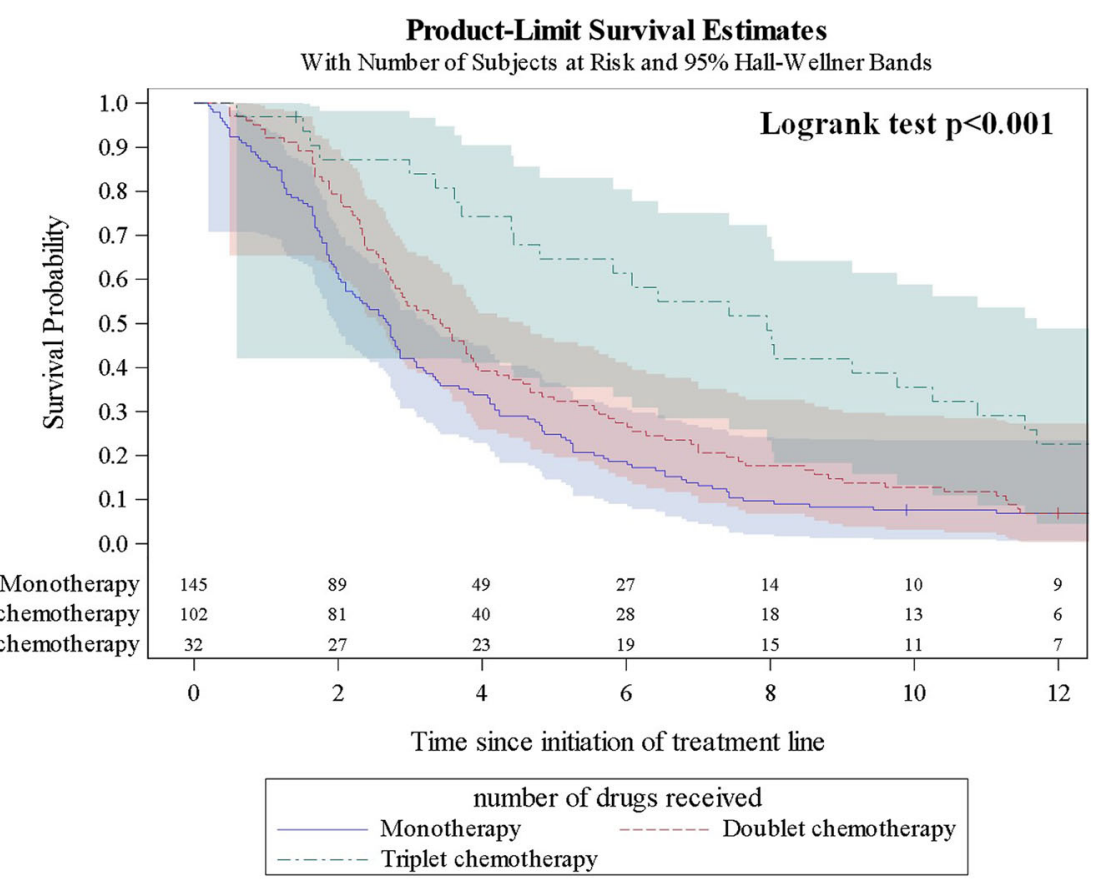

b

Product-Limit Survival Estimates

With Number of Subjects at Risk and $95 \%$ Hall-Wellner Bands

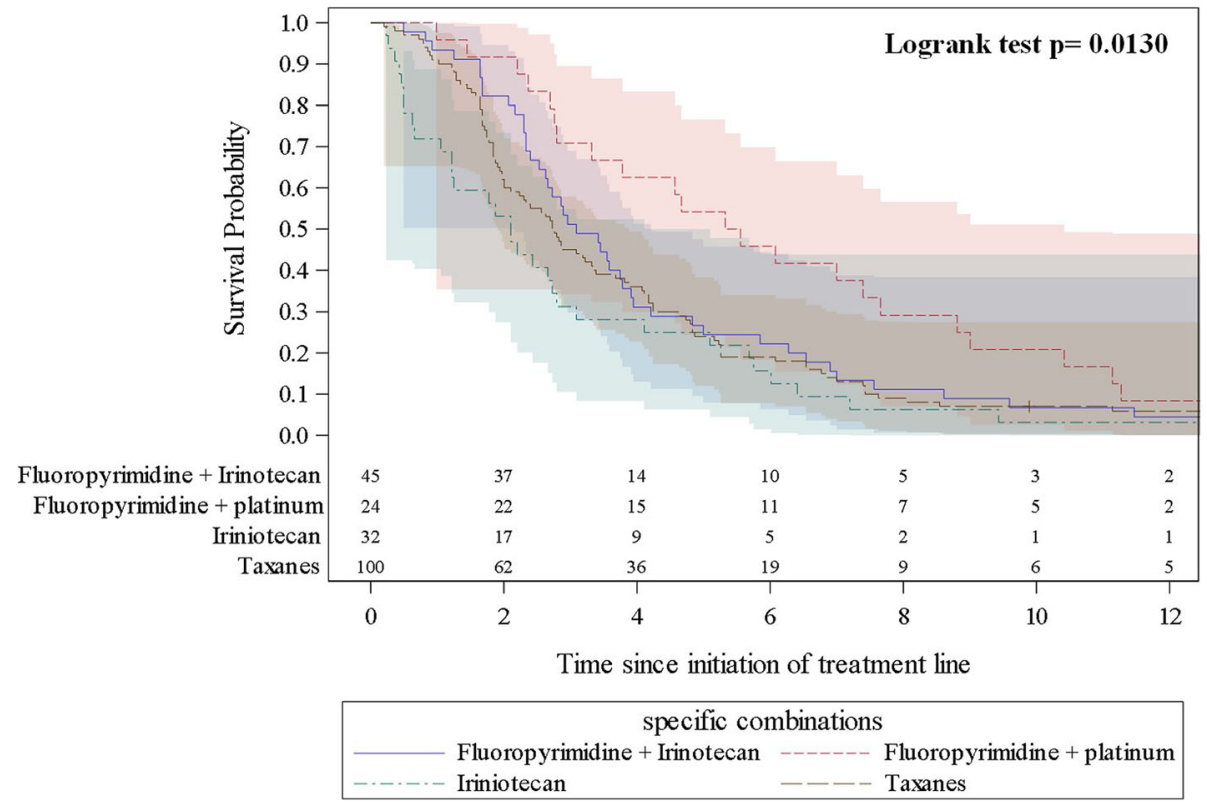

Fig. 2 Progression-free survival from initiation of $2 \mathrm{~L}$ treatment. a PFS with monotherapy, doublet therapy, triplet therapy, and overall; b PFS with taxane monotherapy, irinotecan monotherapy, fluoropyrimidine + irinotecan, and fluoropyrimidine + platinum

24]. Patients included in earlier RCTs, which were the first to provide evidence on the efficacy of $2 \mathrm{~L}$ treatment, were slightly younger, with median ages ranging between 55 and 56 years $[5,6]$.

Only $7.3 \%$ of patients for whom ethnicity was reported were Asian, whereas a number of RCTs have had predominant Asian populations $[6,8-10,23,24]$. In contrast to most RCTs, in which all or the great majority of patients are of good performance status (PS; ECOG 0 or 1$)[6,8-12,23,24]$, approximately $16 \%$ of patients in GENERATE for whom PS data were available had ECOG PS of 2, and 2\% had PS of 3.

Patients in GENERATE were also less likely to have had prior surgery: $15.4 \%$ had surgery for their cancer, 

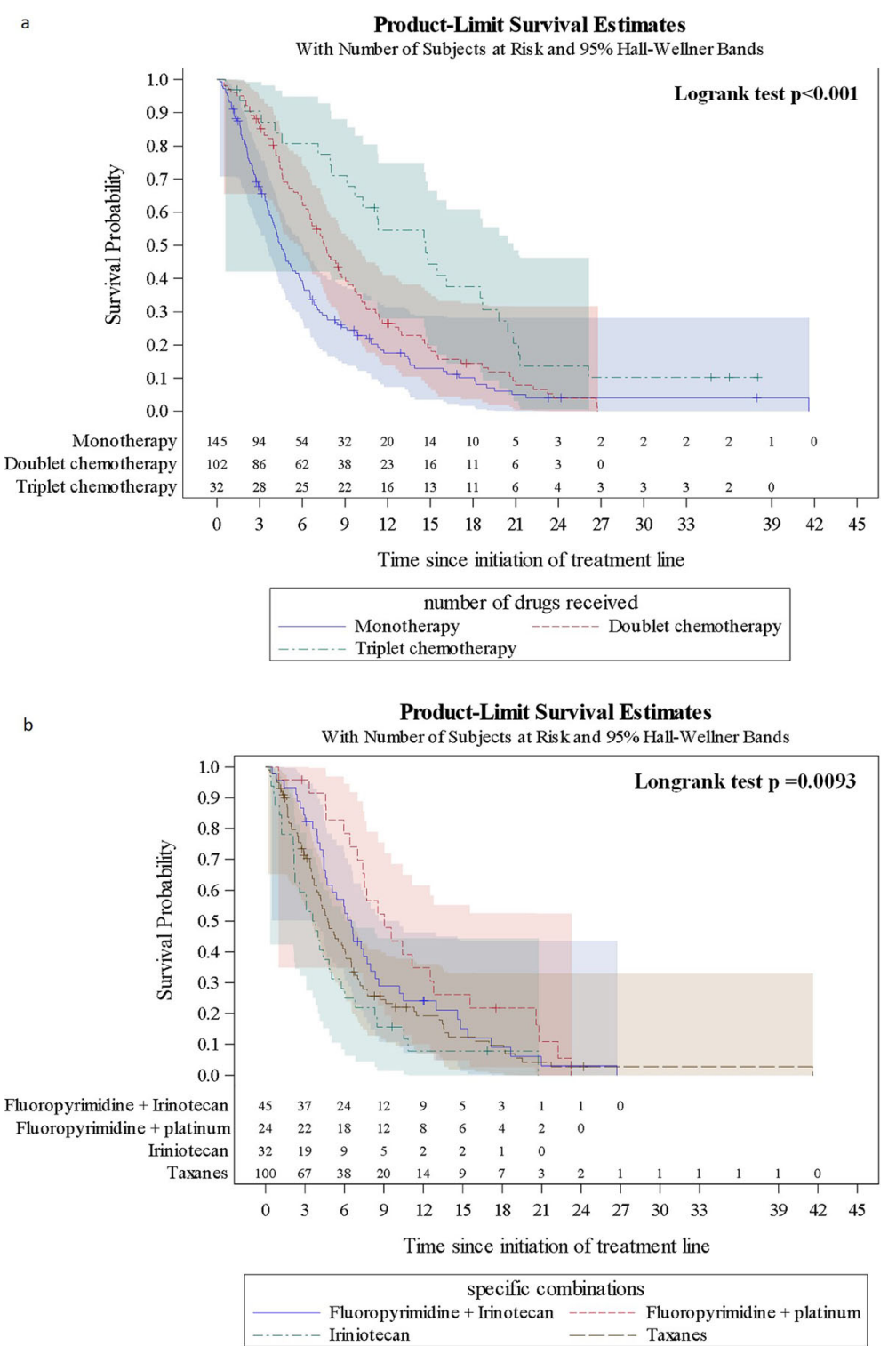

Fig. 3 Overall survival from initiation of $2 \mathrm{~L}$ treatment. a OS with monotherapy, doublet therapy, triplet therapy, and overall; $\mathbf{b}$ OS with taxane monotherapy, irinotecan monotherapy, fluoropyrimidine + irinotecan, fluoropyrimidine + platinum, and overall

compared with $21-58 \%$ in $2 \mathrm{~L}$ RCTs $[5,6,8,9,12,23$, 24]. This might be because they tended to be older and less fit than patients enrolled in RCTs.

Drugs prescribed as $1 \mathrm{~L}$ chemotherapy to patients included in the GENERATE study were also consistent with those reported for patients included in RCTs, with platinum/fluoropyrimidine and taxane/ fluoropyrimidine combinations being the most commonly prescribed. However, there were differences in the proportion of patients who had received triplet vs doublet $1 \mathrm{~L}$ regimens. Those in RCT settings were more likely to have received doublet regimens (60$100 \%$ of patients) than triplet ones $(6-26 \%)[5,6,8$, 9, 11, 12], whereas almost two-thirds of patients in
GENERATE had received fluoropyrimidine/platinum triplet combinations as $1 \mathrm{~L}$ therapy, rather than a doublet. This is unsurprising in the light of evidence demonstrating the significant benefit from addition of an anthracycline to the fluoropyrimidine/platinum regimen [25].

Treatment outcomes reported in GENERATE were also broadly comparable to those reported in RCTs evaluating $2 \mathrm{~L}$ treatments, with modest PFS and OS. For instance, median PFS and OS with irinotecan in an RCT of irinotecan versus BSC were 2.6 and 4.0 months, respectively [5], which are similar to those reported here for irinotecan monotherapy (rwPFS 2.10 months and rwOS 3.70 months). A phase 3 RCT comparing 
Table 4 Number and percentage of patients using each healthcare resource during $2 \mathrm{~L}$ treatment

\begin{tabular}{|c|c|c|c|c|c|}
\hline Resource, n (\%) ${ }^{a}$ & Australia $(N=34)$ & Canada $(N=100)$ & Italy $(N=84)$ & UK $(N=62)$ & Overall $(N=280)$ \\
\hline Hospitalization/inpatient stay & $11(32.4 \%)$ & $30(30.0 \%)$ & $14(16.7 \%)$ & $20(32.3 \%)$ & $75(26.8 \%)$ \\
\hline ICU stay & - & $2(2.0 \%)$ & - & - & $2(0.7 \%)$ \\
\hline Emergency room visit & $3(8.8 \%)$ & 19 (19.0\%) & $2(2.4 \%)$ & $7(11.3 \%)$ & $31(11.1 \%)$ \\
\hline Outpatient (visit for follow-up) & $21(61.8 \%)$ & $27(27.0 \%)$ & $51(60.7 \%)$ & $50(80.6 \%)$ & 149 (53.2\%) \\
\hline Concomitant medication & $30(88.2 \%)$ & $91(91.0 \%)$ & $84(100.0 \%)$ & 60 (96.8\%) & $265(94.6 \%)$ \\
\hline Laboratory tests & $27(79.4 \%)$ & 95 (95.0\%) & 78 (92.9\%) & 61 (98.4\%) & $261(93.2 \%)$ \\
\hline Blood cell count & $27(79.4 \%)$ & $94(94.0 \%)$ & $78(92.9 \%)$ & $61(98.4 \%)$ & $260(92.9 \%)$ \\
\hline Biochemistry test & $27(79.4 \%)$ & $92(92.0 \%)$ & 78 (92.9\%) & $61(98.4 \%)$ & $258(92.1 \%)$ \\
\hline Liver function test & $27(79.4 \%)$ & $92(92.0 \%)$ & 78 (92.9\%) & 57 (91.9\%) & $254(90.7 \%)$ \\
\hline Renal function test & $27(79.4 \%)$ & $94(94.0 \%)$ & $77(91.7 \%)$ & $56(90.3 \%)$ & $254(90.7 \%)$ \\
\hline Blood pressure reading & $26(76.5 \%)$ & $53(53.0 \%)$ & $58(69.0 \%)$ & 49 (79.0\%) & $186(66.4 \%)$ \\
\hline Electrocardiogram & $7(20.6 \%)$ & $18(18.0 \%)$ & $21(25.0 \%)$ & $14(22.6 \%)$ & $60(21.4 \%)$ \\
\hline Imaging tests & $26(76.5 \%)$ & $78(78.0 \%)$ & 37 (44.0\%) & $56(90.3 \%)$ & $197(70.4 \%)$ \\
\hline$X$-ray & $11(32.4 \%)$ & $44(44.0 \%)$ & $17(20.2 \%)$ & $10(16.1 \%)$ & $82(29.3 \%)$ \\
\hline Ultrasound & $8(23.5 \%)$ & $24(24.0 \%)$ & $5(6.0 \%)$ & $9(14.5 \%)$ & $46(16.4 \%)$ \\
\hline CT & $25(73.5 \%)$ & $60(60.0 \%)$ & $27(32.1 \%)$ & $54(87.1 \%)$ & $166(59.3 \%)$ \\
\hline MRI scan & $1(2.9 \%)$ & $5(5.0 \%)$ & $1(1.2 \%)$ & $2(3.2 \%)$ & $9(3.2 \%)$ \\
\hline PET scan & - & $1(1.0 \%)$ & - & $1(1.6 \%)$ & $2(0.7 \%)$ \\
\hline Fluorodeoxyglucose-PET-CT scan & $5(14.7 \%)$ & $2(2.0 \%)$ & $3(3.6 \%)$ & $1(1.6 \%)$ & $11(3.9 \%)$ \\
\hline Endoscopy & $2(5.9 \%)$ & $7(7.0 \%)$ & $2(2.4 \%)$ & $5(8.1 \%)$ & $16(5.7 \%)$ \\
\hline Colonoscopy & - & $1(1.0 \%)$ & $1(1.2 \%)$ & - & $2(0.7 \%)$ \\
\hline
\end{tabular}

${ }^{a}$ number and percentage of patients for whom the resource has been used; denominator is the total number of patients CT Computed tomography, ICU intensive care unit, MRI Magnetic resonance imaging, PET Positron emission tomography

docetaxel with BSC reported a median OS of 5.2 months for docetaxel [7], which is also consistent with the estimated rwOS of 4.7 months for patients treated with taxane monotherapy reported in GENERATE.

More recently, several RCTs have reported efficacy outcomes for PD-1 and PD-1L-targeted immunotherapies in second and subsequent treatment lines. In a phase III RCT comparing pembrolizumab with paclitaxel in $2 \mathrm{~L}$, patients treated with pembrolizumab presented with a median PFS and OS 1.5 and 9.1 months, respectively [26]. In a phase I/II RCT comparing nivolumab monotherapy with two combinations of nivolumab and ipilimumab in patients having received at minimum a first line of treatment, the median PFS and OS for nivolumab monotherapy was 1.4 and 6.2 months, respectively, and ranged between 1.4-1.6 and 4.8-6.9 months, respectively, for the two combination therapy regimens [27]. In the $3 \mathrm{~L}$ setting, the median PFS and OS reported in a phase III RCT that compared avelumab vs physician's choice of chemotherapy was 1.4 and 4.6 months, respectively [28]. These findings place immunotherapy as a potential good alternative for advanced GEJ cancer patients but further studies are awaited to help define these as regular treatment choices for these patients.
Similarly, other novel therapies such as apatinib (rivoceranib) or trifluridine/tipiracil have been recently evaluated in the $3 \mathrm{~L}$ setting. In a double-blind phase III RCT conducted in China comparing apatinib vs. placebo in patients who had failed to at least two previous lines of chemotherapy, the median PFS and OS for apatinib were 2.6 and 6.5 months, respectively [29]. Very recently, results of a global phase III RCT also comparing apatinib vs. placebo indicated that apatinib patents had a median PFS and OS of 2.8 and 5.8 months, respectively although OS was not significantly improved for patients receiving apatinib in $3 \mathrm{~L}$ therapy [30]. In another multi-country doble-blind phase III RCT, that evaluated trifluridine/ tipiracil plus BSC vs. placebo plus BSC, the median PFS and OS were 2.0 and 5.7 months, respectively [31]. These results show the potential to widen the scope of alternative tretments for patients with advanced GEJ cancer, although further studies are warranted to evaluate the potential use of these drugs in the $2 \mathrm{~L}$ setting.

GENERATE found that approximately half of all patients $(51.8 \%)$ had monotherapy in $2 \mathrm{~L}$ treatment, with taxanes (used by $69.0 \%$ of monotherapy patients) and irinotecan $(22.1 \%)$ being the most commonly prescribed monotherapies. This is consistent with evidence showing increased OS with these agents compared with BSC [5- 
7]. Use of ramucirumab was infrequent, reported in 6 patients as monotherapy (4.1\% of monotherapy patients) and 13 patients (9.6\% of combination therapy patients) in combination with taxanes. Almost all of this use was in Italy, as ramucirumab had not yet been approved in Canada or Australia during the study period (index date between January 2013 and July 2015), and although approved by the EMA in December 2014 [32, 33] it was not recommended by NICE in the UK. Use of trastuzumab in $2 \mathrm{~L}$ was also infrequent (16 patients in total, most in combination with taxanes, or as triplet therapy with platinum and fluoropyrimidines).

The findings from GENERATE on treatment patterns are generally similar to those of previous studies evaluating routine clinical practices, although methodological differences between the studies make direct comparisons difficult $[14-18,22]$. A retrospective analysis by Fanatto et al. of 300 patients with advanced or metastatic gastric cancer in Italy who had received at least three chemotherapy regimens between 2000 and 2015 found that for $2 \mathrm{~L}$ therapy, 37.3, 48.7 and $9.7 \%$ of patients were treated with monotherapy, doublet and triplet regimens, respectively [15]. Fluoropyrimidines (52.7\% of patients), taxanes (39.7\%) and irinotecan (39.0\%) were the most commonly prescribed chemotherapy agents in $2 \mathrm{~L}$. [15] Liepa et al. retrospectively reported on 200 patients in the UK dignosed with advanced or metastatic gastric cancer between 2007 and 2012 and who had received $1 \mathrm{~L}$ treatment with a fluoropyrimidine/ platinum regimen, 57 of whom received $2 \mathrm{~L}$ therapy [16]. The lack of a clearly defined standard of care was reflected in the fact that 21 unique $2 \mathrm{~L}$ regimens were reported by Liepa et al., with monotherapy being most common. The most commonly used agents were docetaxel (28\% of patients), paclitaxel $(11 \%)$, trastuzumab $(9 \%)$, capecitabine $(7 \%)$ and irinotecan (7\%) [16]. In a retrospective study by Elsing et al. of 111 patients in Germany who initiated treatment between 2001 and 2011, the most frequently used $2 \mathrm{~L}$ treatment was irinotecan in combination with 5 -FU ( $21 \%$ of patients), and $19 \%$ of patients received irinotecan monotherapy [22]; again, this was similar to the treatment patterns observed in GENERATE. However, Elsing et al. showed that taxane monotherapy was not prescribed as $2 \mathrm{~L}$ treatment, probably because the study included patients who began treatment before evidence on the efficacy of taxanes in $2 \mathrm{~L}$ therapy was published.

In terms of HCRU, the data from GENERATE will be useful to inform economic evaluations of $2 \mathrm{~L}$ treatments for advanced or metastatic gastric cancer, and complement the findings of other studies with different designs and study populations and in different healthcare systems $[14,16,18,34]$. Overall, there was a mean observation period of 6.6 months and approximately a quarter of patients required hospitalization. Admission to ICU was reported in less than $1 \%$ of patients. Emergency room visits were required by over $10 \%$ of patients, whereas outpatient visits were reported by approximately half of patients. Almost all patients received concomitant medications and required laboratory tests, and a high percentage underwent imaging tests (70.4\%). Thus, results from GENERATE indicate substantial resource use for this population.

The study by Liepa et al. [16] conducted in the UK reported that 63,39 and $46 \%$ of patients required followup outpatient visits, emergency room visits and hospitalizations, respectively, but this was measured from the start of $1 \mathrm{~L}$ treatment, rather than the start of $2 \mathrm{~L}$ treatment as in GENERATE. In addition, Liepa et al. excluded outpatient visits related to chemotherapy administration. In GENERATE, more UK patients (80.6\%) had outpatient visits than in Liepa et al., but fewer required emergency room visits (11.3\%) and hospitalization (32.2\%).

Karve et al. reported a retrospective analysis of the Surveillance, Epidemiology, and End Results (SEER)Medicare-linked database that included 2583 patients diagnosed with locally advanced/unresectable or metastatic gastric cancer in the US between 2000 and 2007. Patients were aged $\geq 65$ and had received $1 \mathrm{~L}$ treatment with fluoropyrimidine and/or a platinum chemotherapy agent [14]. In the Karve study, 33.9, 16.8 and $82.5 \%$ of patients who received $2 \mathrm{~L}$ treatment required hospitalizations, emergency room visits and outpatient visits, respectively. The proportions of patients requiring hospitalizations and emergency room visits were relatively similar to those observed in GENERATE (which were 26.8 and $11.1 \%$, respectively), whereas the proportion requiring outpatient visits in GENERATE was much lower (53.2\%). This may be explained by the longer observation period in the study conducted by Karve et al.

There are several strengths associated with the GENERATE study. Eligible patients had to have been followed at the participating site and have clinical chart data available for the entire observation period, thus enabling a comprehesive and longitudinal picture of treatment and HRCU during $2 \mathrm{~L}$ therapy. Detailed information on treatment patterns, HCRU and outcomes was collected for patients in whom the full medical history was available, from initiation of $2 \mathrm{~L}$ treatment and for a 12-month follow up, which would cover the whole treatment line in most cases. Participating physicians were all experienced in the management of gastric cancer.

A few study limitations need to be noted. The study was based on retrospective chart review, which may be 
associated with systematic under-recording of some types of information on the clinical charts. Secondly, assessment of disease progression was based exclusively on evaluation by the treating physician, and may have been influenced by local diagnostic practices and treatment standards. There may also have been inconsistencies in the time points at which disease progression was assessed. Thirdly, the study data were collected from a convenience sample of sites that routinely manage patients with advanced or metastatic gastric cancer, and may not be representative of all sites in the respective countries. Finally, the real burden of the management of advanced gastric cancer patients may be understimated, as the use of healthcare resources associated with palliative care was not specifically captured in this study.

\section{Conclusions}

An increasing number of studies have shown $2 \mathrm{~L}$ treatment for advanced or metastatic gastric cancer to be effective and feasible. However, despite recent evidence, there are still no clear recommendations established across clinical guidelines to enable an understanding of current treatment patterns. To this end, the GENERATE study adds to the literature by providing insights on patient characteristics, treatment patterns, HCRU and clinical outcomes of real-world patients diagnosed with advanced or metastatic gastric or GEJ adenocarcinoma who initiated $2 \mathrm{~L}$ therapy between January 2013 and July 2015 across four different countries (Australia, Canada, Italy and the UK). Further work, including the collection of more recent data and the collection of data on palliative care, would further clarify contemporary treatment patterns in this setting and help understand the burden of lost lifes.

\section{Supplementary information}

Supplementary information accompanies this paper at https://doi.org/10. 1186/s12876-020-01232-z.

\section{Additional file 1: Table S1. Drugs received in $2 L$ chemotherapy.}

Table S2. Drugs received in $3 \mathrm{~L}$ chemotherapy. Table S3. Overall survival multivariate analysis results (excluding patients with ECOG PS missing). Table S4. Progression-free survival multivariate analysis results (excluding patients with ECOG PS missing).

\section{Abbreviations \\ $1 \mathrm{~L}$ : First line; $2 \mathrm{~L}$ : Second line; $3 \mathrm{~L}$ : Third line; AE: Adverse event; BSC: best supportive care; ECOG: Eastern Cooperative Oncology Group Performance Status test; GEJ: Gastroesophageal junction; HCRU: Healthcare resource utilization; HER2: Human epidermal growth factor receptor 2; ICU: Intensive care unit; OS: Overall survival; PFS: Progression free survival; RCT: Randomized controlled trials; rwOS: Real-world overall survival; rwPFS: Real-world progression free survival; UK: United Kingdom}

\section{Acknowledgements}

The authors would like to acknowledge the contribution of all investigators, sites and patients who participated in this research. We also acknowledge the contribution of Ceylan Jasari from the CORE Operations Study
Management group at Merck \& Co., Inc., Kenilworth, NJ, USA, for her operational and project management support. Finally, we would like to thank Montse Roset and Elisabet Viayna from IQVIA for their contribution in the conduct of statistical analysis and medical writing, respectively.

\section{Authors' contributions}

DG and NL substantially contributed to the design of the study, the analysis and interpretation of data and the drafting of the manuscript. MA and SK substantially contributed to the conception and design of the study, and the interpretation of data. WC, IC, JZ and AF substantially contributed to the design of the study, and the interpretation of data. All authors have contributed, read and approved the final manuscript.

\section{Funding}

This work was financially supported by Merck Sharp \& Dohme Corp., a subsidiary of Merck \& Co., Inc., Kenilworth, NJ, USA (MSD). MSD acted as study sponsor by funding the coordination of all study-related activities and by providing fair market value grants to the participating sites, related to study set-up and patient inclusion.

\section{Availability of data and materials}

The datasets used and analysed during the current study are available from the corresponding author on reasonable request.

\section{Ethics approval and consent to participate}

All procedures followed were in accordance with the ethical standards of the responsible committee on human experimentation (institutional and national) and with the Helsinki Declaration of 1964 and later versions. This study has received ethical approval from the Medical Ethical Committee of the participating sites. The participant hospitals and medical institutions are: Royal Marsden NHS foundation Trust of Fullham Road, Christie NHS foundation trust of Wilmslow Road, University Hospital Bristol NHS Trust of Horfield Road, Maidstone and Tumbridge Wells NHS Trust of Hermitage Lane, East Lancashire Hospital HNS Trust of Haslingden Road, Azienda sanitaria locale Lecce, Azienda sanitaria locale Napoliznord, Azienda Ospidaliera Arnas Garibaldi, Azienda Sanitaria Universitaria Integrada de Udine, Azienda Ospedaliera Universitaria di Pisa, Azienda ULSS, Ospedale di Cremona, Cliniche Gavazzeni SpA, Tom Barker Centre, Sir Mortimer B. Davis Jewish General Hospital, Ottawa Hospital Research Institute, Nova Scotia Health Authority, Sinai Health System, Southern Medical Day Care Centre, St John of God Murdoch Hospital, Sunshine Hospital, Ballarat Base Hospital, Westmead Hospital, St Vincent's Health Network. A written informed consent or the equivalent was obtained from all patients, unless waived by the corresponding Ethical Committee. This article does not contain any studies with animal subjects performed by any of the authors.

Consent for publication

Not applicable.

\section{Competing interests}

DG reports that IQVIA, employer of DG, received consulting fees from Merck \& Co, Inc. during the conduct of the study. MA reports being a full time employee and stockholder of Merck \& Co. Inc. during the conduct of the study. SK reports being a full time employee of Merck \& Co. Inc. during the conduct of the study. IC reports personal fees from Merck Sharp \& Dohme Corp. during the conduct of the study; grants, personal fees and advisory board fees from Eli-Lilly, personal fees from Bristol Meyer Squibb, personal fees from Bayer, grants and personal fees from Merck Serono, personal fees from Roche, personal fees from Astra-Zeneca, grants from Janssen-Cilag, grants from Sanofi, fees from Five Prime Therapeutics, personal fees from Oncologie International, outside the submitted work. JZ reports grants and personal fees from Bayer, personal fees and travel support from Merck \& Co. Inc., personal fees and travel support from Merck Sharp \& Dohme Corp, outside the submitted work. NL reports that IQVIA, employer of NL, received consulting fees from Merck \& $\mathrm{Co}$, Inc. during the conduct of the study. AF reports personal fees from Servier during the conduct of the study; grants and personal fees from Roche, grants and personal fees from Bayer, grants and personal fees from Merck \& Co. Inc., grants and personal fees from Amgen, personal fees from Servier, grants from Merck Sharp \& Dohme Corp, personal fees from Bristol, personal fees from Lilly, outside the submitted work. All remaining authors have no conflicts of interest to declare. 


\section{Author details}

${ }^{1}$ Real World Solutions, IQVIA, Barcelona, Spain. ${ }^{2}$ Merck \& Co., Inc., North Wales, PA, USA. ${ }^{3}$ Merck \& Co., Inc., Kenilworth, NJ, USA. ${ }^{4}$ British Columbia Cancer Agency, Vancouver, BC, Canada. ${ }^{5}$ Royal Marsden Hospital, London \& Surrey, UK. ${ }^{6}$ Alfred Health and School of Public Health, Monash University, Melbourne, Australia. ${ }^{7}$ University of Pisa, Pisa, Italy.

Received: 12 September 2019 Accepted: 24 March 2020

Published online: 05 May 2020

\section{References}

1. Bray F, Ferlay J, Soerjomataram I, Siegel RL, Torre LA, Jemal A. Global cancer statistics 2018: GLOBOCAN estimates of incidence and mortality worldwide for 36 cancers in 185 countries. CA Cancer J Clin. 2018;68(6):394-424.

2. Guggenheim DE, Shah MA. Gastric cancer epidemiology and risk factors. J Surg Oncol. 2012;107(3):230-6.

3. Bang Y-J, Yalcin S, Roth A, Hitier S, Ter-Ovanesov M, Wu C-W, Zalcberg J. Registry of gastric cancer treatment evaluation (REGATE): I baseline disease characteristics. Asia-Pacific J Clin Oncol. 2013;10(1):38-52.

4. Wagner AD, Grothe W, Haerting J, Kleber G, Grothey A, Fleig WE. Chemotherapy in advanced gastric Cancer: a systematic review and metaanalysis based on aggregate data. J Clin Oncol. 2006;24(18):2903-9.

5. Thuss-Patience PC, Kretzschmar A, Bichev D, Deist T, Hinke A, Breithaupt K, Dogan Y, Gebauer B, Schumacher G, Reichardt P. Survival advantage for irinotecan versus best supportive care as second-line chemotherapy in gastric cancer - a randomised phase III study of the Arbeitsgemeinschaft Internistische Onkologie (AIO). Eur J Cancer. 2011;47(15):2306-14.

6. Kang JH, Lee SI, Lim DH, Park KW, Oh SY, Kwon HC, Hwang IG, Lee SC, Nam E, Shin DB, et al. Salvage chemotherapy for pretreated gastric cancer: a randomized phase III trial comparing chemotherapy plus best supportive care with best supportive care alone. J Clin Oncol. 2012;30(13):1513-8.

7. Ford HER, Marshall A, Bridgewater JA, Janowitz T, Coxon FY, Wadsley J, Mansoor W, Fyfe D, Madhusudan S, Middleton GW, et al. Docetaxel versus active symptom control for refractory oesophagogastric adenocarcinoma (COUGAR-02): an open-label, phase 3 randomised controlled trial. Lancet Oncol. 2014;15(1):78-86.

8. Hironaka S, Ueda S, Yasui H, Nishina T, Tsuda M, Tsumura T, Sugimoto N, Shimodaira H, Tokunaga S, Moriwaki T, et al. Randomized, open-label, phase III study comparing Irinotecan with paclitaxel in patients with advanced gastric Cancer without severe peritoneal metastasis after failure of prior combination chemotherapy using Fluoropyrimidine plus platinum: WJOG 4007 trial. J Clin Oncol. 2013;31(35):4438-44.

9. Higuchi K, Tanabe S, Shimada K, Hosaka H, Sasaki E, Nakayama N, Takeda Y, Moriwaki T, Amagai K, Sekikawa T, et al. Biweekly irinotecan plus cisplatin versus irinotecan alone as second-line treatment for advanced gastric cancer: a randomised phase III trial (TCOG GI-0801/BIRIP trial). Eur J Cancer. 2014;50(8):1437-45.

10. Nishikawa K, Fujitani K, Inagaki H, Akamaru Y, Tokunaga S, Takagi M, Tamura S, Sugimoto N, Shigematsu T, Yoshikawa T, et al. Randomised phase III trial of second-line irinotecan plus cisplatin versus irinotecan alone in patients with advanced gastric cancer refractory to S-1 monotherapy: TRICS trial. Eur J Cancer. 2015;51(7):808-16.

11. Fuchs CS, Tomasek J, Yong CJ, Dumitru F, Passalacqua R, Goswami C, Safran H, dos Santos LV, Aprile G, Ferry DR, et al. Ramucirumab monotherapy for previously treated advanced gastric or gastro-oesophageal junction adenocarcinoma (REGARD): an international, randomised, multicentre, placebo-controlled, phase 3 trial. Lancet. 2014;383(9911):31-9.

12. Wilke H, Muro K, Van Cutsem E, Oh S-C, Bodoky G, Shimada Y, Hironaka S, Sugimoto N, Lipatov O, Kim T-Y, et al. Ramucirumab plus paclitaxel versus placebo plus paclitaxel in patients with previously treated advanced gastric or gastro-oesophageal junction adenocarcinoma (RAINBOW): a doubleblind, randomised phase 3 trial. Lancet Oncol. 2014;15(11):1224-35.

13. Salati M, Di Emidio K, Tarantino V, Cascinu S. Second-line treatments: moving towards an opportunity to improve survival in advanced gastric cancer? ESMO Open. 2017;2(3):e000206.

14. Karve S, Lorenzo M, Liepa AM, Hess LM, Kaye JA, Calingaert B. Treatment patterns, costs, and survival among Medicare-enrolled elderly patients diagnosed with advanced stage gastric Cancer: analysis of a linked population-based Cancer registry and administrative claims database. J Gastric Cancer. 2015;15(2):87.
15. Fanotto V, Uccello M, Pecora I, Rimassa L, Leone F, Rosati G, Santini D, Giampieri R, Di Donato S, Tomasello G, et al. Outcomes of advanced gastric Cancer patients treated with at least three lines of systemic chemotherapy. Oncologist. 2017;22(12):1463-9.

16. Liepa AM, Brown J, Bapat B, Kaye JA. Real-world treatment patterns of previously treated advanced gastric and gastroesophageal junction adenocarcinoma (GC) in the United Kingdom (UK). J Clin Oncol. 2015; 33(3_suppl):184.

17. Rha SY, Calvo E, Contente M, Rider A, Maglinte G, Clarke B, Muro K. 208PReal-world advanced gastric cancer treatment patterns in Western and Asian countries. Ann Oncol. 2017;28(suppl_10):mdx660.015.

18. Carter GC, Kaltenboeck A, Ivanova J, Liepa AM, San Roman A, Koh M, Rajan $\mathrm{N}$, Cheng R, Birnbaum HG, Kim JS, et al. Real-world treatment patterns among patients with advanced gastric Cancer in South Korea. Cancer Res Treat. 2017:49(3):578-87.

19. Sherman KL, Merkow RP, Shah AM, Wang CE, Bilimoria KY, Bentrem DJ. Assessment of advanced gastric Cancer Management in the United States. Ann Surg Oncol. 2013;20(7):2124-31.

20. Mahar AL, Coburn NG, Viola R, Johnson AP. Predictors of hospital stay and home care services use: a population-based, retrospective cohort study in stage IV gastric cancer. Palliat Med. 2014;29(2):147-56.

21. Monti M, Foca F, Gardini AC, Valgiusti M, Frassineti GL, Amadori D. Retrospective analysis on the Management of Metastatic Gastric Cancer Patients. A mono-institutional experience. What happens in clinical practice? Tumori J. 2013;99(5):583-8.

22. Elsing C, Herrmann C, Hannig CV, Stremmel W, Jäger D, Herrmann T. Sequential chemotherapies for advanced gastric Cancer: a retrospective analysis of 111 patients. Oncology. 2013;85(5):262-8.

23. Ohtsu A, Ajani JA, Bai Y-X, Bang Y-J, Chung H-C, Pan H-M, Sahmoud T, Shen $\mathrm{L}$, Yeh $\mathrm{K}-\mathrm{H}$, Chin $\mathrm{K}$, et al. Everolimus for previously treated advanced gastric Cancer: results of the randomized, double-blind, phase III GRANITE-1 study. J Clin Oncol. 2013;31(31):3935-43.

24. Satoh T, Xu R-H, Chung HC, Sun G-P, Doi T, Xu J-M, Tsuji A, Omuro Y, Li J, Wang J-W, et al. Lapatinib plus paclitaxel versus paclitaxel alone in the second-line treatment ofHER2-amplified advanced gastric Cancer in Asian populations: TyTAN_A randomized, phase III study. J Clin Oncol. 2014; 32(19):2039-49.

25. Okines AFC, Norman AR, McCloud P, Kang YK, Cunningham D. Meta-analysis of the REAL-2 and ML17032 trials: evaluating capecitabine-based combination chemotherapy and infused 5-fluorouracil-based combination chemotherapy for the treatment of advanced oesophago-gastric cancer. Ann Oncol. 2009;20(9):1529-34.

26. Shitara K, Ozguroglu M, Bang YJ, Di Bartolomeo M, Mandala M, Ryu MH, Fornaro L, Olesinski T, Caglevic C, Chung HC, et al. Pembrolizumab versus paclitaxel for previously treated, advanced gastric or gastro-oesophageal junction cancer (KEYNOTE-061): a randomised, open-label, controlled, phase 3 trial. Lancet (London, England). 2018;392(10142):123-33.

27. Janjigian $Y Y$, Bendell J, Calvo E, Kim JW, Ascierto PA, Sharma P, Ott PA, Peltola K, Jaeger D, Evans J, et al. CheckMate-032 study: efficacy and safety of Nivolumab and Nivolumab plus Ipilimumab in patients with metastatic Esophagogastric Cancer. J Clin Oncol. 2018;36(28):2836-44..

28. Bang YJ, Ruiz EY, Van Cutsem E, Lee KW, Wyrwicz L, Schenker M, Alsina M, Ryu $\mathrm{MH}$, Chung $\mathrm{HC}$, Evesque $\mathrm{L}$, et al. Phase III, randomised trial of avelumab versus physician's choice of chemotherapy as third-line treatment of patients with advanced gastric or gastro-oesophageal junction cancer: primary analysis of JAVELIN gastric 300. Ann Oncol. 2018;29(10):2052-60.

29. Li J, Qin S, Xu J, Xiong J, Wu C, Bai Y, Liu W, Tong J, Liu Y, Xu R, et al. Randomized, double-blind, placebo-controlled phase III trial of Apatinib in patients with chemotherapy-refractory advanced or metastatic adenocarcinoma of the stomach or Gastroesophageal junction. J Clin Oncol. 2016;34(13):1448-54.

30. Kang YK, Kang WK, Di Bartolomeo M, Chau I, Yoon HH, Cascinu S, Ryu MH, Kim JG, Lee KW, Oh SC, et al. Randomized phase III ANGEL study of rivoceranib (apatinib) + best supportive care (BSC) vs placebo + BSC in patients with advanced/metastatic gastric cancer who failed \&\#x2265;2 prior chemotherapy regimens. Ann Oncol. 2019;30:v877-8.

31. Shitara K, Doi T, Dvorkin M, Mansoor W, Arkenau HT, Prokharau A, Alsina M, Ghidini M, Faustino C, Gorbunova V, et al. Trifluridine/tipiracil versus placebo in patients with heavily pretreated metastatic gastric cancer (TAGS): a randomised, double-blind, placebo-controlled, phase 3 trial. Lancet Oncol. 2018;19(11):1437-48 
32. Cyramza. Product monograph. Lilly Canada Available at: http://www.lilly.ca/ en/pdf/product-monograph/cyramza-ca-pm.pdf (Accessed 19 Feb 2018).

33. European Medicines Agency Committee for Medicinal Products for Human Use (CHMP). Summary of opinion. Cyramza. EMA/CHMP/552028/2014.; 2014

34. Cuyun Carter G, Kaltenboeck A, Ivanova J, Liepa AM, San Roman A, Koh M, Rajan N, Cheng R, Birnbaum H, Chen J-S. Treatment patterns in patients with advanced gastric cancer in Taiwan. Asia-Pacific J Clin Oncol. 2016;13(3): 185-94.

\section{Publisher's Note}

Springer Nature remains neutral with regard to jurisdictional claims in published maps and institutional affiliations.

Ready to submit your research? Choose BMC and benefit from:

- fast, convenient online submission

- thorough peer review by experienced researchers in your field

- rapid publication on acceptance

- support for research data, including large and complex data types

- gold Open Access which fosters wider collaboration and increased citations

- maximum visibility for your research: over $100 \mathrm{M}$ website views per year

At $\mathrm{BMC}$, research is always in progress.

Learn more biomedcentral.com/submissions 